\title{
OPEN Oxidative stress and motion sickness in one crew during competitive offshore sailing
}

\author{
Tommaso Antonio Giacon ${ }^{1 凶}$, Gerardo Bosco ${ }^{1 凶}$, Alessandra Vezzoli², Cinzia Dellanoce ${ }^{2}$,
} Danilo Cialoni ${ }^{1}$, Matteo Paganini ${ }^{1}$ \& Simona Mrakic-Sposta ${ }^{2}$

Competitive Offshore Ocean Sailing is a highly demanding activity in which subjects are exposed to psychophysical stressors for a long time. To better define the physiological adaptations, we investigated the stress response of subjects exposed to 3-days long ocean navigation with disruption of circadian rhythms. 6 male subjects were involved in the study and provided urine and saliva samples before setting sail, during a single day of inshore sailing, during 3-days long ocean navigation, and at the arrival, to measure oxidative stress, cortisol, nitric oxide metabolites (NOX) and metabolic response. Motion Sickness questionnaires were also administered during the navigation. The crew suffered a mean weight loss of $1.58 \mathrm{~kg}$. After the long navigation, a significant increase in ROS production and decrease in total antioxidant capacity and uric acid levels were observed. Lipid peroxidation, NO metabolites, ketones, creatinine, and neopterin levels were also increased. Furthermore, a significant increase in cortisol levels was measured. Finally, we found a correlation between motion sickness questionnaires with the increase of NOx, and no correlation with cortisol levels. Physical and psychological stress response derived from offshore sailing resulted in increased oxidative stress, nitric oxide metabolites, and cortisol levels, unbalanced redox status, transient renal function impairment, and ketosis. A direct correlation between motion sickness symptoms evaluated through questionnaires and NOx levels was also found.

$\begin{array}{ll}\begin{array}{l}\text { Abbreviations } \\ \text { 8-iso-PGF2 } \alpha\end{array} & \text { 8-Isoprostane } \\ \text { EPR } & \text { Electron paramagnetic resonance } \\ \text { GERD } & \text { Gastroesophageal reflux disease } \\ \text { GSRS } & \text { Global Sickness Rating Scale } \\ \text { MSQ } & \text { Motion Sickness Questionnaire } \\ \text { NO } & \text { Nitric oxide } \\ \text { NOx }\left(\mathrm{NO}_{2}+\mathrm{NO}_{3}\right) & \text { Nitric oxide metabolites } \\ \text { OOS } & \text { Offshore ocean sailing } \\ \text { OxS } & \text { Oxidative stress } \\ \text { ROS } & \text { Reactive oxygen species } \\ \text { SWH } & \text { Significant wave height } \\ \text { TAC } & \text { Total antioxidant capacity } \\ \text { TWA } & \text { True wind angle }\end{array}$

Sailing is a worldwide popular activity that includes various types of boats and disciplines. Offshore Ocean Sailing (OOS) could represent a relaxing leisure activity, but in case of competitions and regattas it is considered one of the most extreme endurance sports, exposing the crew to long-lasting, physically and psychologically demanding efforts ${ }^{1}$. Competitive OOS usually implies prolonged periods-ranging from days to months-spent at sea, in an extremely harsh environment, in isolation and self-sufficiency, far from safe harbours and with limited access to external aid or rescue ${ }^{2}$. The boat could represent an extremely uncomfortable, cold, wet, unstable, and enclosed environment without any privacy or comfort. In particular, racing boats are performance-oriented and with little or no comfort onboard. Energy expenditure during offshore sailing is high $^{3}$, and it is challenging to

\footnotetext{
${ }^{1}$ Department of Biomedical Sciences, Environmental and Respiratory Physiology, University of Padova, Via Marzolo 3, 35131 Padua, Italy. ${ }^{2}$ Institute of Clinical Physiology, National Research Council (CNR), Milan, Italy. ${ }^{\boxplus}$ email: tommasoantonio.giacon@studenti.unipd.it; gerardo.bosco@unipd.it
} 
maintain an adequate nutrient intake onboard, especially during harsh weather conditions ${ }^{4}$. Negative energy balance often results in weight loss, decreased body fat percentage, and reduced muscle strength, proportionally to the length of the race ${ }^{5,6}$. Proper sleep management is also essential to maintain adequate performance ${ }^{7}$. Sailors adopt polyphasic sleep techniques and incur severe sleep restrictions during competition, thus resulting in cognitive performance and alertness decrease ${ }^{8}$.

The study of adaptations to extreme environments is gaining popularity. Nonetheless, the literature exploring short- and medium-term adaptations to OOS is still insufficient. Seafarers are also exposed to high and prolonged stress levels. Loneliness, circadian rhythms disruption, and fatigue often result in alterations in their physical ${ }^{9}$ and psychological domains ${ }^{10}$. Consistently, high-stress levels can induce a modification in normal circadian fluctuations of cortisol, a glucocorticoid whose peak level in normal conditions is recorded after awakening ${ }^{11}$. During OOS and other highly stressing activities, a flattening of this curve has been recorded, with sustained high cortisol levels throughout the effort ${ }^{12}$. Due to the constant instability, the maritime environment significantly impacts cognitive and neuromuscular activity ${ }^{13}$. Moreover, motion sickness often affects people exposed to transportation and visual instability. The underlying cause is still ambiguous, some theories address a possible sensory mismatch mechanism between perceived and expected stimuli ${ }^{14}$. Others ascribe it to situations in which we are not able to maintain postural stability such as during maritime navigations ${ }^{15}$. A large percentage of people experience seasickness, with higher work-related risks and detrimental effects on the performance of sailors and seafarers ${ }^{14,16}$.

During inshore and offshore sailing, physical effort is inconstant, characterized by high intensity and anaerobic bursts, with increases in oxygen consumption and heart rate ${ }^{1,17-19}$. Such activity often leads to heat loss and dehydration $^{5,20}$, and produces muscle damage and oxidative stress (OxS). OxS levels have been investigated in other endurance sports, such as triathlon ${ }^{21}$, ultra-endurance races $^{22}$, and swimming ${ }^{23}$, revealing an overproduction of Reactive Oxygen Species (ROS) and a depletion of total antioxidant capacity (TAC). The redox status-namely, the equilibrium between ROS and TAC-deeply affects intracellular function. Maintaining ROS homeostasis is crucial for normal cellular responses, while overproduction is deleterious and can damage cell structures (i.e., proteins, membrane, DNA), leading to progressive organism's disfunction ${ }^{24,25}$. Along with ROS production, increased levels of nitrogen metabolites and in particular nitric oxide (NO), a crucial messenger in many tissues such as endothelium and gastrointestinal tissues, can be found under stressful condition ${ }^{26}$. Nonetheless, a formal assessment in sailing sports and specifically during OOS is still lacking.

This study aimed to investigate oxidative stress variations in sailors involved in OOS. To have a more accurate definition of stress, we also evaluated cortisol levels, biochemical profile, and renal function markers creatinine and neopterin. Motion sickness has been investigated through neurophysiological symptoms questionnaires.

\section{Methods}

Experimental design. This observational study was carried on in November 2020 during an OOS racingoriented training that included a theoretical part ashore, a full training day of inshore sailing, and 3 days of nonstop OOS roughly between the latitudes of Gibraltar and Lisbon. The crew sailed in a Class 40 (ITA 84) racing yacht and, during the navigation, was divided into two groups alternating rest and duty shifts every 3 h. Figure 1 depicts the study protocol and samplings. Urine and saliva samples and anthropometric measurements were obtained ashore during the theoretical part (PRE) and approximately two hours after the end of the navigation (POST) to be as accurate as possible. Further, two urine samples were obtained during the single day of inshore navigation (Training) and three times a day during OOS (Sailing).

Subjects. This study involved six male sailors: the skipper (S.F.) - a professional sailor with experience in ocean solo races-and five recreational sailors with good expertise in seamanship The total number of subjects was determined by the maximum crew that the boat could support and by chance all the participants to this training were healthy male. Their characteristics are reported in Table 1.

Navigation. The offshore navigation lasted 3 days, during which the crew sailed into the ocean for a total of 420 miles, with a top speed of $14.89 \mathrm{kn}$.

During the first day and night, the atmospheric conditions were challenging. Swell of $3 / 3.5 \mathrm{~m}$ Significant Wave Height (SWH) from NW and wind from 5 to $15 \mathrm{kn}$ from S-SE resulted in a boat's inconvenient motion. During the day, the wind increased between 25 and $40 \mathrm{kn}$ in gusts as sailors encountered two significant squalls and had to flee downwind. During the night, sailors were forced to maintain a $70^{\circ}$ true wind angle (TWA) sailing upwind to cope with waves, and the wind speed increased up to $45 \mathrm{kn}$. After the first day, the crew was objectively stressed. These harsh conditions induced major seasickness and vomiting in one crew member, with a total inability to work on deck. This subject started to recover only at the end of the navigation, during which he never ate and vomited many times without being able to drink and rehydrate. Two other people vomited but were not impaired at work. Liquid reintegration started the day after. On the second day, the conditions changed, with waves height reduced to 1-2 $\mathrm{m} \mathrm{SWH}$. The boat headed downwind, hoisting a code zero sail, maintaining the boat flat at an average speed of 9-10 kn with $15-25 \mathrm{kn}$ of wind speed. The navigation remained stable until the end of the navigation in Lisbon on the third day.

Motion Sickness Questionnaire. To study motion sickness, previously validated Global Sickness Rating Scale (GSRS) ${ }^{27}$ and Motion Sickness Questionnaire (MSQ) ${ }^{14}$ were used. They have been administered once per day during the Offshore Sailing, in the evening, at 9 PM, according to the $3 \mathrm{~h}$ shifts. 


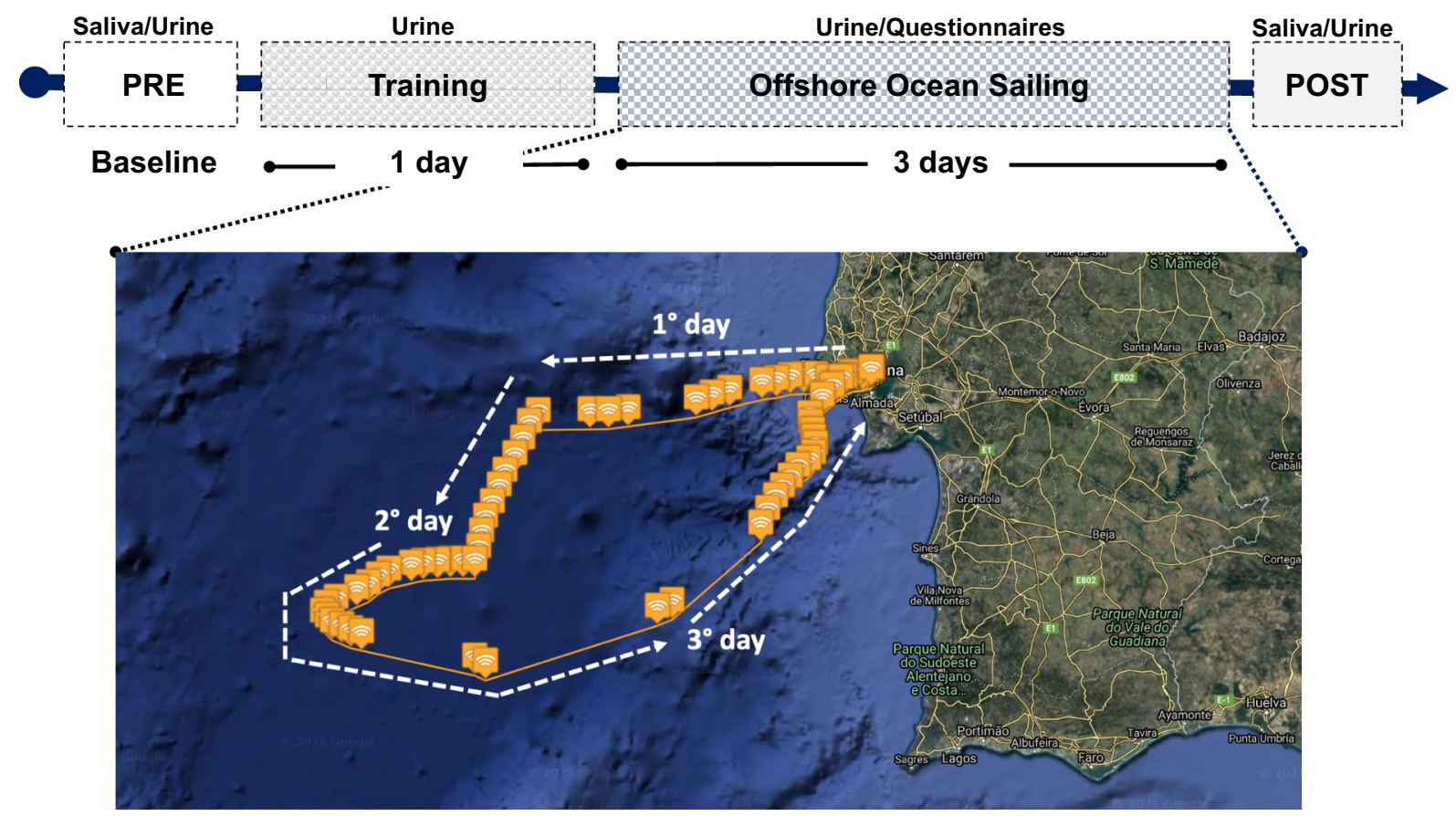

Figure 1. Sketch of the experimental protocol and map showing the navigation route. Data were collected before departure (PRE), during the inshore navigation (Training) during each day of Offshore Ocean Sailing (from $1^{\circ}$ to $3^{\circ}$ day), and at arrival (POST). GPS data and map were obtained with Spot Gen3, SPOT LCC, Globastar, Inc, Covington, Louisiana, USA. Maps by Google, Inst. Geogr. Nacional. Modified geographic map was edited with Microsoft Office PowerPoint 2007, Microsoft Corporation, Washington, USA, version 16.16.27, https://www.microsoft.com/it-it/microsoft-365/powerpoint and at the top was insert the experimental protocol timeline.

\begin{tabular}{|l|l|l|l|l|l|l|}
\hline & Age & Height $(\mathbf{c m})$ & $\begin{array}{l}\text { Weight }(\mathrm{kg}) \\
\text { PRE }\end{array}$ & $\begin{array}{l}\text { Weight }(\mathrm{kg}) \\
\text { POST }\end{array}$ & $\begin{array}{l}\text { BMI }\left(\mathbf{k g ~ m}^{-2}\right) \\
\text { PRE }\end{array}$ & $\begin{array}{l}\text { BMI }\left(\mathbf{k g ~ m}^{-2}\right) \\
\text { POST }\end{array}$ \\
\hline 1 & 25 & 170 & 65.2 & 63 & 22.6 & 21.8 \\
\hline 2 & 41 & 180 & 86.2 & 84.5 & 26.6 & 26.0 \\
\hline 3 & 31 & 185 & 93.3 & 90.2 & 27.2 & 26.4 \\
\hline 4 & 51 & 187 & 125.2 & 123.1 & 35.8 & 35.2 \\
\hline 5 & 36 & 184 & 83.6 & 83.5 & 24.6 & 24.6 \\
\hline 6 & 26 & 177 & 84.2 & 83.1 & 26.9 & 26.5 \\
\hline Mean \pm SD & $35.16 \pm 9.70$ & $180.50 \pm 6.28$ & $89.62 \pm 19.76$ & $87.90 \pm 19.59^{*}$ & $27.28 \pm 4.52$ & $26.75 \pm 4.50$ \\
\hline
\end{tabular}

Table 1. Anthropometric parameters of the six sailors. Parameters collected from the sailors at Pre and Post. $B M I$ Body Mass Index. ${ }^{\star} \mathrm{p}<0.05$.

Saliva and urine collection. Approximately $1 \mathrm{~mL}$ of saliva was obtained before and after the training and collected in Salivette devices (Sarstedt, Nümbrecht, Germany) at 8 AM. The subjects were trained on the correct use as previously reported ${ }^{28,29}$.

Urine samples were collected by voluntary voiding in a sterile container before and after the training and every day during the training and navigation at $9 \mathrm{AM}, 3 \mathrm{PM}$, and $9 \mathrm{PM}$ according to the $3 \mathrm{~h}$ shifts. All samples were stored at $4{ }^{\circ} \mathrm{C}$ in a portable cooler on board and during the transport back to the laboratory. The specimens were then stored in multiple aliquots at $-20^{\circ} \mathrm{C}$ until assayed and thawed only once before analysis.

ROS and TAC. An X-band electron paramagnetic resonance spectroscopy $(9.3 \mathrm{GHz})$ (E-Scan, Bruker Co., MA, USA) was used to detect ROS production and TAC values. Saliva samples were stabilized at $37^{\circ} \mathrm{C}$ using a Temperature and Gas Controller "Bio III" unit (Noxigen Science Transfer \& Diagnostics GmbH, Germany), interfaced with the E-Scan. ROS production and TAC assessment methods were previously described ${ }^{28,30}$. Samples were analyzed in triplicate.

Cortisol. The concentration of free cortisol in the saliva was quantitatively determined through ELISA method according to the manufacturer's protocol (COR(Cortisol) ELISA Kit; FineTest, Wuhan Fine Biotech Co.) as previously described ${ }^{31}$. 
8-Isoprostane. Lipid peroxidation was assessed in urine by competitive immunoassay measuring 8-isoprostane concentration (8-iso-PGF2 $\alpha$ ) (Cayman Chemical, USA). The method was previously described ${ }^{32}$.

NO metabolites. NO metabolites $\left(\mathrm{NOx}=\mathrm{NO}_{2}+\mathrm{NO}_{3}\right)$ levels were assessed in urine by a method based on the Griess reaction ${ }^{33}$, using a commercial kit (Cayman, BertinPharma, Montigny le Bretonneux, France) ${ }^{28,33}$.

Every assessment was carried out in duplicate and read by a microplate reader spectrophotometer (Infinite M200, Tecan Group Ltd., Männedorf, Switzerland).

Creatinine, neopterin, and uric acid. Urinary creatinine, neopterin, and uric acid concentrations were measured by isocratic high-pressure liquid chromatography. The calibration curves were linear over the range of 0.125-1 $\mu \mathrm{mol} / \mathrm{L}, 0.625-20 \mathrm{mmol} / \mathrm{L}$, and $1.25-10 \mathrm{mmol} / \mathrm{L}$ for neopterin, uric acid, and creatinine levels, respectively. Inter-assay and intra-assay coefficients of variation were $<5 \%$. Methods were previously described ${ }^{28,34}$.

Urine standard analysis. The Urine Test Strips (Combi screen 11sys PLUS, GIMA, Gessate, Milan, Italy) were used to semi-quantitative determinations of bilirubin, urobilinogen, ketones, proteins, blood, pH, leukocytes, and specific gravity/density in urine. The tests were performed in duplicate.

Statistical analysis. Statistical analysis was performed using the GraphPad Prism package (GraphPad Prism 9.0.1, GraphPad Software Inc., San Diego, CA) and SPSS statistics software (IBM corporation). Data are presented as mean \pm SD. Statistical analyses were performed using: non-parametric tests, Wilcoxon matchedpairs signed-rank test for independent samples (ROS and TAC and cortisol in saliva), due to the small sample size for compared pre vs. post and ANOVA repeated measures, with Dunn's multiple comparison tests to further check the among-groups significance. $\mathrm{p}<0.05$ was considered statistically significant..dCohen was used for calculating the size effect, and Confidence Interval $95 \%$ for $d_{\text {Cohen }}$ was calculated.

Change $\Delta \%$ estimation $[(($ post value-pre value $) /$ pre-value $) \times 100]$ is also reported in the text. Spearman correlation ( $r)$ with $95 \%$ confidence intervals. Chi-square $\left(\chi^{2}\right)$ test and phi coefficient $(\phi)$ were used to evaluate correlation and association.

Ethics approval. This research study was approved by the Ethical Committee of University of Milan, Italy (Aut. $n^{\circ} 37 / 17$ ). All procedures conformed to the standards set by the 1964 Declaration of Helsinki and its later amendments.

Consent to participate. Informed consent was obtained from all individual participants included in the study.

Consent for publication. All authors have read the manuscript and expressed their consent for the publication.

\section{Results}

A significant difference $(\mathrm{p}<0.05)$ was observed in weight $(\mathrm{kg})$ between Pre and Post (Table 1$)$. All crew members suffered a loss of weight (Mean weight loss: $1.58 \mathrm{~kg}$ ).

An unbalance of oxidative stress was found. ROS production rate in saliva significantly $(\mathrm{p}<0.01)$ increased at Post OOS $\left(0.27 \pm 0.07\right.$ vs $0.54 \pm 0.16 \mu \mathrm{mol} \mathrm{min}^{-1}$, dCohen $=0.74$; $95 \%$ CI $1.171-4.326$; Fig. $\left.2 \mathrm{~A}\right)$ with a significant decrease $(\mathrm{p}<0.05)$ in antioxidant capacity (TAC $2.50 \pm 0.19$ vs $2.21 \pm 0.16 \mathrm{mM}$, dCohen $=1.65 ; 95 \%$ CI 2.961-0.341; Fig. 2B). In addition, uric acid measured in urines significantly decreased (range $\mathrm{p}<0.05-0.001)$ during sailing ( $8.61 \pm 3.74$ vs. $4.28 \pm 1.66$ vs. $3.18 \pm 1.36 \mathrm{mM}$ at Post, dCohen $=1.49,95 \%$ CI $2.777-0.216$; and $\mathrm{dCohen}=1.93,95 \%$ CI 3.3-0.56 respectively; Fig. 2C). A significant increase (range $\mathrm{p}<0.05-0.001)$ in lipid peroxidation during OOS (8-isoprostane $228.40 \pm 63.1$ vs $378.68 \pm 103.69 \mathrm{pg} \mathrm{mg}^{-1}$ creatinine; dCohen $=1.75$, 95\% CI 0.42-3.081) and at Post OOS (8-isoprostane $427.70 \pm 134.98 \mathrm{pg} \mathrm{mg}^{-1}$ creatinine, dCohen $=1.89,95 \%$ CI 0.53-3.253; Fig. 2D) was measured; besides NO metabolites significantly (range p $<0.05-0.001$ ) increased during OOS (NOx 331.8 \pm 102.2 vs $504.5 \pm 94.85 \mu \mathrm{M}$; dCohen $=1.75,95 \%$ CI $0.422-3.085$ ) and at Post (NOx $331.8 \pm 102.2$ vs $623.0 \pm 68.24 \mu \mathrm{M}$; dCohen $=3.35,95 \%$ CI $1.656-5.218)$ (Fig. $2 \mathrm{E}$ ).

The time course in Fig. 2F, G showed a significant increase post OOS ( $\mathrm{p}<0.05-0.01)$ of creatinine $(1.57 \pm 0.31$ vs. $1.77 \pm 0.52$ vs. $3.38 \pm 1.01 \mathrm{~g} \mathrm{~L}^{-1}$; dCohen $\left.=2.43,95 \% \mathrm{CI} 0.938-3.922\right)$ and neopterin/creatinine $(70.92 \pm 11.80$ vs. $105.603 \pm 22.14$ vs. $23.33 \pm 35.25 \mu \mathrm{mol} \mathrm{mol}^{-1}$ creatinine: dCohen $=1.99,95 \%$ CI $0.609-3.377$, and dCohen $=1.95,95 \%$ CI $0.579-3.33$ ) levels respectively at sailing and post OOS.

Finally, a significant increase $(\mathrm{p}<0.05)$ in cortisol levels was measured Post OOS in saliva $(2.47 \pm 0.36$ vs. $2.85 \pm 0.22 \mathrm{ng} / \mathrm{mL}^{-1}$, dCohen $=1.27,95 \%$ CI $0.033-2.515$; Fig. $\left.2 \mathrm{H}\right)$.

No significant differences were recorded in GSRS for different items and MSQ during the 3 days of offshore navigation (see Tables 2, 3).

Finally, a positive relationship was found during navigation (training and sailing) between NO metabolites and Global Sickness Rating Scale (GSRS) scores $(r=0.94, p<0.05)$ (Fig. 3A); between GSRS score and specific items of Motion Sickness Questionnaire (MSQ), in details: during the 1st day with General discomfort $r=0.83$ $(\mathrm{p}=0.04)$ (Fig. 3B), during 2nd day with General discomfort $\mathrm{r}=0.87(\mathrm{p}=0.02)$ (Fig. 3C), dizziness as illusory sense of motion (eyes open) $r=0.85(p=0.03)$ (Fig. 3D), and stomach awareness $r=0.98(p=0.0007)$ (Fig. 3E); during the 3 rd day no correlation was found. 

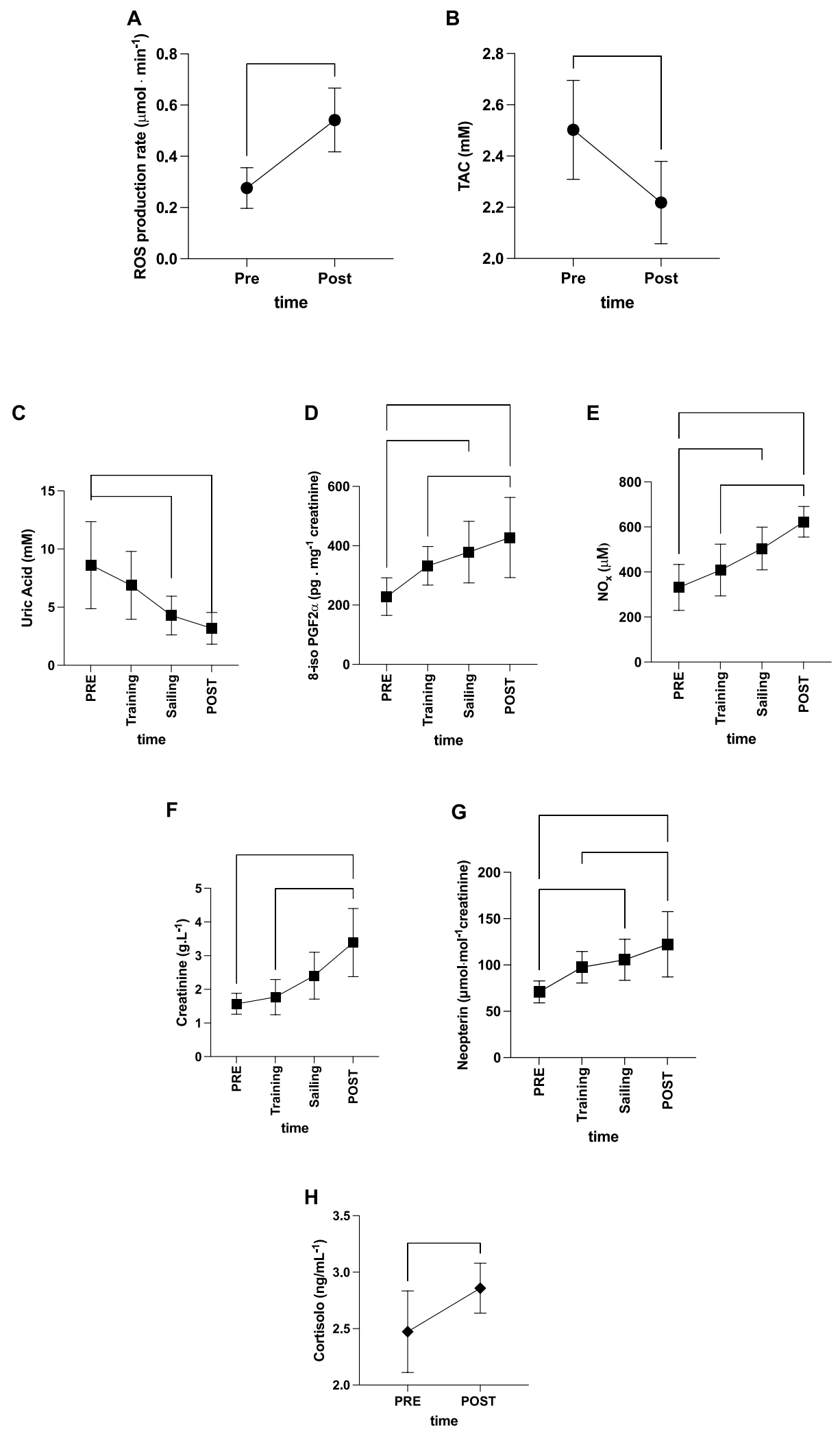

Figure 2. Biomarkers kinetic. Time course of: (A) radical oxygen species (ROS) production rate $\left(\mu \mathrm{mol} \cdot \mathrm{min}^{-1}\right)$ and (B) total antioxidant capacity (TAC-mM) in saliva assessed by EPR; (C) uric acid (mM); (D) 8-isoprostane (8-iso-PGF2 $\alpha, \mathrm{pg} \mathrm{mg}^{-1}$ creatinine); (E) nitric oxide metabolites (NOx, $\left.\mu \mathrm{M}\right)$, (F) creatinine $\left(\mathrm{g} \mathrm{L}^{-1}\right),(\mathbf{G})$ neopterin ( $\mu \mathrm{mol} \mathrm{mol}{ }^{-1}$ creatinine), concentrations detected in urine. In $(\mathbf{H})$ cortisol levels $(\mathrm{ng} / \mathrm{mL})$ measured in saliva. ${ }^{*} \mathrm{p}<0.05,{ }^{* *} \mathrm{p}<0.01,{ }^{* *} \mathrm{p}<0.001$ significantly different. Figure created with: GraphPad Prism, GraphPad Software inc. California, USA, version 9.0.1, https://www.graphpad.com/. 


\begin{tabular}{|l|l|l|l|}
\hline \multirow{2}{*}{ Sailors (n=6) } & \multicolumn{3}{l}{ Global Sickness Rating } \\
Scale (GSRS) \\
\hline Scores & Day 1 & Day 2 & Day 3 \\
\hline 1: No symptoms & $\mathrm{n} 2(2)$ & $\mathrm{n} 2(2)$ & $\mathrm{n} 5(5)$ \\
\hline 2: Initial symptoms of motion sickness but no nausea & - & $\mathrm{n} 1(2)$ & - \\
\hline 3: Mild Nausea & $\mathrm{n} 1(3)$ & - & $\mathrm{n} 1(3)$ \\
\hline 4: Moderate Nausea & - & $\mathrm{n} 1(4)$ & - \\
\hline 5: Severe nausea and/or retching & - & $\mathrm{n} 1(5)$ & - \\
\hline 6: Vomiting & $\mathrm{n} 3(18)$ & $\mathrm{n} 1(6)$ & - \\
\hline Total score & $23 \pm 3.8$ & $19 \pm 2.1$ & $8 \pm 0.8$ \\
\hline
\end{tabular}

Table 2. Global Sickness Rating Scale (GSRS), number of subjects and (total value) are reported for each day. Total scores \pm SD are reported.

\begin{tabular}{|l|c|l|l|}
\hline \multirow{2}{*}{ Sailors (n=6) } & \multicolumn{3}{l}{ Motion Sickness Questionnaire } \\
(MSQ) & \multicolumn{2}{l|}{} \\
\cline { 2 - 4 } & Day 1 & Day 2 & Day 3 \\
\hline General discomfort & $1.0 \pm 1.2$ & $1.33 \pm 1.03$ & $0.83 \pm 0.72$ \\
\hline Fatigue & $1.16 \pm 0.98$ & $1.16 \pm 0.98$ & $1.33 \pm 0.81$ \\
\hline Headache & $0.83 \pm 1.16$ & $0.66 \pm 1.21$ & $0.33 \pm 0.51$ \\
\hline Eye Strain & $0.16 \pm 0.40$ & $0.16 \pm 0.40$ & $0.33 \pm 0.51$ \\
\hline Difficulty focusing & $0.16 \pm 0.40$ & $0.0 \pm 0.0$ & $0.16 \pm 0.40$ \\
\hline Increased salivation & $0.0 \pm 0.0$ & $0.0 \pm 0.0$ & $0.16 \pm 0.40$ \\
\hline Fulness of head & $0.80 \pm 1.16$ & $0.83 \pm 0.51$ & $0.33 \pm 0.51$ \\
\hline Blurred vision & $0.0 \pm 0.0$ & $0.0 \pm 0.0$ & $0.0 \pm 0.0$ \\
\hline Dizziness as illusory sense of motion (eyes open) & $0.33 \pm 0.51$ & $0.33 \pm 0.51$ & $0.0 \pm 0.0$ \\
\hline Dizziness as illusory sense of motion (eyes closed) & $0.33 \pm 0.81$ & $0.66 \pm 1.21$ & $0.16 \pm 0.40$ \\
\hline Vertigo & $0.50 \pm 0.83$ & $1.33 \pm 1.21$ & $0.16 \pm 0.40$ \\
\hline Stomach awareness & $1.50 \pm 1.22$ & $1.33 \pm 1.21$ & $0.83 \pm 0.75$ \\
\hline Burping & $1.50 \pm 1.22$ & $0.66 \pm 0.81$ & $0.16 \pm 0.40$ \\
\hline
\end{tabular}

Table 3. Motion Sickness Questionnaire (MSQ). Mean $( \pm S D)$ values of the investigated variables. Urine standard parameters are reported in Table 4. A significant increase in urinary ketones levels was detected during the navigation. $\mathrm{pH}$ and bilirubin values also increased but did not reach statistical significance.

\section{Discussion}

To our knowledge, this is the first study to investigate oxidative stress on urine and saliva sampled from nonprofessional sailors during OOS and possible correlation with motion sickness. This setting is particularly challenging, with rapid changes in terms of environmental conditions and circadian rhythms.

According to the results, subjects exposed to OOS suffer a significant multifactorial increase in oxidative stress and cortisol. A small number of studies considered modifications in cortisol levels in sailors and seafarers. Oldenburg et al. found that cortisol awakening levels were highly dependent on subjective stress perception and work type. Mental work was also associated with higher cortisol levels than physical work ${ }^{35}$. This is confirmed by cortisol levels found in maritime pilots, increasing their tasks' difficulty ${ }^{36}$. Some studies' results reflect that seafarers' cortisol levels are higher in port stays than at sea, probably because of the break of a working routine found during days at sea ${ }^{35,37}$. Liberzon et al. found that cortisol response at awakening in the crew increased with navigation time and was not correlated with sleep duration or patterns ${ }^{37}$. Other confined environments in which the crew suffers a sudden and prolonged change in circadian rhythms and psychosocial stress are, for example, military ships, submarines, or spaceships. In these environments, a flattening of the standard cortisol fluctuation profile has also been recorded under stressful conditions ${ }^{38,39}$. Similar results were obtained by Gunnarsson et al. on ocean sailors studied for a more extended period during an offshore regatta. They also reported an initial increase in cortisol levels at the beginning of the navigation, with a flattening of the fluctuation when sailors reached the regularization of the shift regimen ${ }^{12}$. Our study found a significant increase in cortisol levels in sailors after 3-day-long offshore navigation compared to their basal level (+15\%, see Fig. $2 \mathrm{H})$. These results may be related to the significant stressors that maritime personnel and offshore ocean sailors have to endure, particularly fatigue and poor sleep quality. From a physiological point of view, sleep disturbances induce a decrease in physical and cognitive performance in sailors ${ }^{8}$ and a disruption of normal cortisol secretion, causing the activation of pro-inflammatory pathways ${ }^{40,41}$. Physical exercise can also induce a modification in cortisol secretion ${ }^{42}$. During offshore sailing, a basal level of muscle activation is needed to cope with instability and to aid 


\begin{tabular}{|c|c|c|c|c|c|}
\hline \multicolumn{6}{|l|}{ Sailors $n=6$} \\
\hline & PRE & Day 1 & Day 2 & Day 3 & POST \\
\hline Bilirubin $\left(\mu \mathrm{mol} \mathrm{L}{ }^{-1}\right)$ & $5.65 \pm 8.80$ & $6.16 \pm 9.55$ & $11.66 \pm 9.07$ & $17.00 \pm 1.09$ & $11.16 \pm 8.75$ \\
\hline Urobilinogen $\left(\mu \mathrm{mol} \mathrm{L}^{-1}\right)$ & $0.2 \pm 0.1$ & $0.2 \pm 0.1$ & $0.2 \pm 0.1$ & $0.2 \pm 0.1$ & $0.2 \pm 0.1$ \\
\hline Ketones $\left(\mathrm{mmol} \mathrm{L}^{-1}\right)$ & $1.0 \pm 2.23$ & $1.0 \pm 2.0$ & $16.16 \pm 18.49^{*}$ & $12.16 \pm 14.2^{\star}$ & $9.0 \pm 5.47^{*}$ \\
\hline Protein $\left(\mathrm{mg} \mathrm{dL}^{-1}\right)$ & $0.0 \pm 0.0$ & $0.0 \pm 0.0$ & $0.0 \pm 0.0$ & $0.0 \pm 0.0$ & $0.0 \pm 0.0$ \\
\hline Blood $\left(\right.$ Ery $\left.\mu \mathrm{L}^{-1}\right)$ & $0.0 \pm 0.0$ & $0.0 \pm 0.0$ & $0.0 \pm 0.0$ & $0.0 \pm 0.0$ & $0.0 \pm 0.0$ \\
\hline $\mathrm{pH}$ & $5.16 \pm 0.40$ & $6.50 \pm 1.25$ & $6.12 \pm 1.43$ & $6.25 \pm 1.04$ & $5.87 \pm 1.18$ \\
\hline Leucocytes (Leuko $\left.\mu \mathrm{L}^{-1}\right)$ & $0.0 \pm 0.0$ & $0.0 \pm 0.0$ & $0.0 \pm 0.0$ & $0.0 \pm 0.0$ & $0.0 \pm 0.0$ \\
\hline Specific gravity/density & $1.01 \pm 0.00$ & $1.02 \pm 0.00$ & $1.03 \pm 0.00^{*}$ & $1.03 \pm 0.00^{*}$ & $1.03 \pm 0.00^{*}$ \\
\hline
\end{tabular}

Table 4. Urine standard analysis. Mean $( \pm S D)$ values of the investigated variables in the urine test strip in the sailors. Changes in urine standard urinalysis referred to PRE are shown. Statistically significant difference at $\mathrm{p}<0.05\left({ }^{*}\right.$ symbol).

A

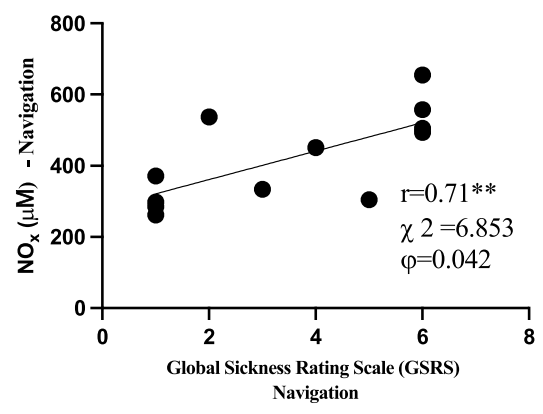

B

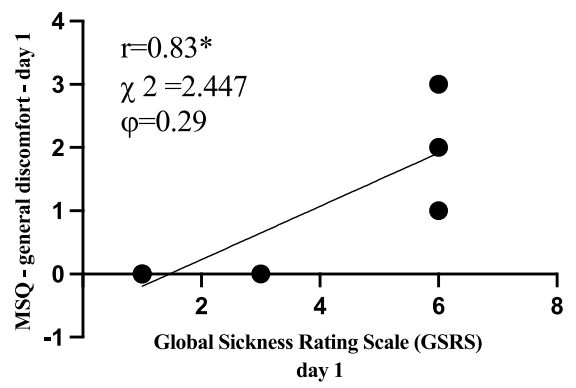

C

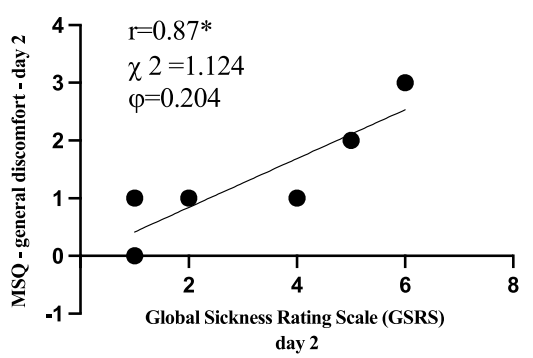

D

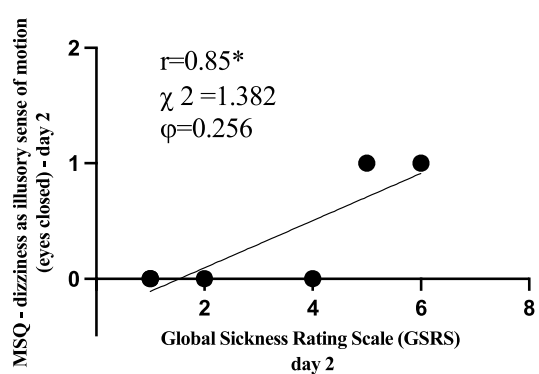

E

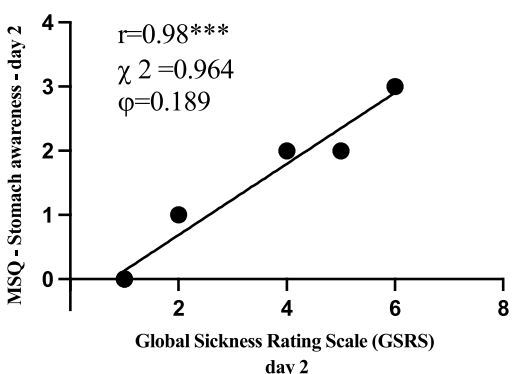

Figure 3. Relationship panel plot of: (A) NOx and GSRS during navigation; (B) GSRS and general discomfort of MSQ at 1st day; at the 2nd day, the panels show the correlation between GSRS and specific items of MSQ. (C) General discomfort and GSRS. (D) Dizziness as illusory sense of motion (eyes open) and GSRS. (E) Stomach awareness and GSRS. A significant linear relationship $(\mathrm{p}<0.05-0.001)$ between parameters was estimated. The correlation coefficient $(r)$, Chi-Square $\left(\chi^{2}\right)$ and Phi coefficient $(\phi)$ are reported. Figure created with: GraphPad Prism, GraphPad Software inc. California, USA, version 9.0.1, https://www.graphpad.com/.

thermoregulation, but short bursts of anaerobic exercise are required in all the maneuvers ${ }^{1,17-19}$. It is, therefore, reasonable to think that these aspects also contribute to cortisol levels alterations.

During day one, three subjects suffered from motion sickness and had vomiting episodes. Although cortisol is known to correlate with acute nausea and vomiting ${ }^{43,44}$, we found no significant correlation between this hormone and the seasickness scale questionnaires administered ${ }^{14,27}$. Nonetheless, we have been able to measure nitric oxide metabolites (NOx) levels throughout the navigation. Nitric oxide is involved in many gastrointestinal mucosal mechanisms ${ }^{26}$, and previous studies found a correlation between salivary and serum NO levels with vomiting syndromes and Gastroesophageal Reflux Disease (GERD) ${ }^{43-46}$. Nausea caused by motion sickness is also characterized by gastric dysrhythmias ${ }^{47}$. In accordance with these studies, we found a significant linear relationship between NOx levels and GSRS during the first day of navigation, during which the subjects suffered the most intense motion sickness (Fig. 3). 
During inshore regattas, short bursts of high-intensity activity are described ${ }^{18,19,48}$. However, even if data regarding physical effort during offshore sailing seem comparable with inshore activity ${ }^{17}$, the evidence is scarce, difficult to obtain, and limited to measuring the effects of energy expenditure and physical effort activity after the race. Weight loss, fat percentage decrease, lower limb strength, and muscle mass reduction are often reported ${ }^{1,3,4,6,49}$. ROS production is enhanced by exercise $\mathrm{e}^{21-23,29,50}$. In particular, anaerobic exercise can induce prolonged oxidative stress up to $24 \mathrm{~h}$ after the effort ${ }^{51,52}$, which is then balanced by an enhanced antioxidant response $e^{21,23,53}$. In America's Cup sailors, oxidative stress markers after the race were higher than their baseline levels, especially in crew members involved in high-intensity physical work ${ }^{54}$. Our study is the first to analyze oxidative stress markers during OOS. Our results show a significant increase in ROS production after the navigation. The imbalance between the ROS production rate (about $+100 \%)$ and the antioxidant scavenging $(-12 \%$, see Fig. $2 \mathrm{~A}-\mathrm{C}$ ) reflected the increase in the oxidative stress-related damage to lipids (+87\%; see Fig. 2D). Oxidative stress is highly involved with inflammation and endothelial dysfunction in developing chronic cardiovascular disease $^{55,56}$. Even though more evidence should be produced on ocean sailors, the effects of oxidative stress exposition on seafarers can be a potential cause of their higher cardiovascular risk and mortality rate for coronary heart disease $\mathrm{e}^{57-59}$.

Neopterin and creatinine concentration can increase during systemic oxidative stress, as shown in some studies ${ }^{23,28,29,60}$. Even if a decrease in kidney function can be a hint of organ damage during endurance sports, it is often the result of many physiological responses to stress and physical demands ${ }^{21,22,61}$. In our study, an increase of evaluated biomarkers concentrations was observed during and post-offshore sailing and was associated with ROS production. In any case, this study did not assess the chronic or long-term effects of offshore sailing. Mainly referred to kidney activity, the subjects manifested a temporary "impairment of renal function" as a likely physiological or adaptive response to dehydration. This could also be linked to significant weight loss (see Table 1) and vomiting, which changes ketones concentration, $\mathrm{pH}$, and specific density (see Table 4). Ketones increase could also hint at how athletes' metabolism copes with high energy demand and stress. Their production is stimulated by low insulin, high glucagon, and epinephrine concentrations, suggesting a shift to metabolic efficiency and fuel sparing of the organism exposed to endurance exercise and fasting ${ }^{62,63}$. They are also second messengers for many pathways, such as food intake stimuli ${ }^{64}$. The ketogenic regimen is also related to the increase of lipid metabolism ${ }^{65}$ which in the case of OOS is often associated with the decrease of body fat percentage and weight $\operatorname{loss}^{4,6,49}$. Considering that sailors are exposed to harsh environmental conditions and that motion sickness and working rates can influence nutrition habits during a race ${ }^{4}$, it is of utmost importance to maintain an adequate water intake during navigation to prevent renal damage and to keep proper caloric intake to sustain physical performance.

\section{Limitations and conclusions}

As for other studies ${ }^{4,6,12,17}$ that focus on OOS, we found many difficulties in producing reliable data and scientific evidence. The researcher himself, which was part of the crew, had to take part in the strenuous activity schedule, the space for medical devices and samples on board is limited, invasive procedures are complicated to perform because of continuous motion, electronic devices cannot be charged because electrical power is limited and needed for navigation. The complexity of this environment often results in a lack of reliable literature ${ }^{1}$. Therefore, we chose to obtain urine and saliva samples because of the limited logistic disadvantages of these samples.

A limitation of this study is the lack of data on the quality and duration of sleep. This might have influenced the cortisol level, but Liberzon et al. found no connection between sleep and cortisol levels ${ }^{37}$, and other OOS experiments show data similar to ours ${ }^{12}$.

Some previous experiences in literature ${ }^{66}$ found a correlation between cortisol levels and motion sickness. We speculate that probably due to the low number of investigated subjects and saliva samples, and because we didn't measure cortisol levels immediately after vomiting episodes, we found no significant correlation between motion sickness questionnaire results and cortisol levels. Therefore, to be more confident on these results our methods could be implemented. Another factor to be considered could be that we investigated only male subjects, and as previously reported by Meissner et al., the cortisol level changes in saliva in male patients could not be significant. Cortisol response in motion sickness, as they suggest, should be corrected for the hour of the day, gender, and basal cortisol levels ${ }^{67}$. Moreover, high variability was observed in oxidative stress markers, cortisol levels, and motion sickness scales between the same sailors on various days.

Another limitation is that we have not been able to obtain information on sailors' cardiovascular and metabolic activity during the navigation, even though they have been described in other similar and comparable studies $^{17,18}$. In the future, we hope we will be able to implement our methods and obtain this data in a similar environment.

However, the present offshore sailing study offers valuable information on the redox state, renal function, and motion sickness response during this high demanding activity. OOS has been shown to induce an increase in oxidative stress biomarkers and NO metabolites. A correlation was found also between the increase in NO metabolites level and motion sickness intensity evaluated through questionnaires and symptoms. In this experiment, a transient reduction in renal function was found. Moreover, salivary cortisol increased in response to physical activity and stress induced by navigation. Future studies are required to investigate the biochemical processes and the clinical correlations consequent to maritime exposure.

\section{Data availability}

The datasets generated and analyzed during the current study are available from the corresponding author on reasonable request. 
Received: 24 June 2021; Accepted: 10 January 2022

Published online: 21 January 2022

\section{References}

1. Allen, J. B. \& De Jong, M. R. Sailing and sports medicine: A literature review. Commentary. Br. J. Sports Med. 40(7), 587-593 (2006).

2. Sjøgaard, G., Inglés, E. \& Narici, M. Science in sailing: Interdisciplinary perspectives in optimizing sailing performance. Eur. J. Sport Sci. 15(3), 191-194. https://doi.org/10.1080/17461391.2015.1008583 (2015).

3. Myers, S. D., Leamon, S. M., Nevola, V. R. \& Llewellyn, M. G. L. Energy expenditure during a single-handed transatlantic yacht race. Br. J. Sports Med. 42(4), 285-288 (2008).

4. Fearnley, D. et al. Case study of a female ocean racer: Prerace preparation and nutritional intake during the Vendée Globe 2008. Int. J. Sport Nutr. Exerc. Metab. 22(3), 212-219 (2012).

5. Bigard, A. \& Guillemot, P. C. J. Y. Nutrient intake of elite sailors during a solitary long-distance offshore race. Int. J. Sport Nutr. 8 , 364-376 (1998).

6. Lafère, P., Gatzoff, Y., Guerrero, F., Provyn, S. \& Balestra, C. Field study of anthropomorphic and muscle performance changes among elite skippers following a transoceanic race. Int. Marit. Health 71(1), 20-27 (2020).

7. Léger, D. et al. Sleep management and the performance of eight sailors in the Tour de France à la voile yacht race. J. Sports Sci. 26(1), 21-28 (2008).

8. Hurdiel, R. et al. Sleep restriction and degraded reaction-time performance in Figaro solo sailing races. J. Sports Sci. 32(2), 172-174 (2014).

9. Oldenburg, M., Hogan, B. \& Jensen, H. J. Systematic review of maritime field studies about stress and strain in seafaring. Int. Arch. Occup. Environ. Health 86(1), 1-15 (2013).

10. Carotenuto, A., Molino, I., Fasanaro, A. M. \& Amenta, F. Psychological stress in seafarers: A review. Int. Marit. Health 63(4), 188-194 (2012).

11. Stalder, T. et al. Assessment of the cortisol awakening response: Expert consensus guidelines. Psychoneuroendocrinology 63, 414432. https://doi.org/10.1016/.psyneuen.2015.10.010 (2016).

12. Gunnarsson, L. G., Bäck, H., Jones, I. \& Olsson, T. Stress recovery during an ocean boat race. Stress Health 20(3), 165-171 (2004).

13. Pisula, P. J., Lewis, C. H. \& Bridger, R. S. Vessel motion thresholds for maintaining physical and cognitive performance: A study of naval personnel at sea. Ergonomics 55(6), 636-649 (2012).

14. Golding, J. F. Motion sickness [Internet], Handbook of Clinical Neurology, Vol 137 1st edn, 371-390 (Elsevier, 2016). https://doi. org/10.1016/B978-0-444-63437-5.00027-3.

15. Stoffregen, T. A., Chen, F. C., Varlet, M., Alcantara, C. \& Bardy, B. G. Getting your sea legs. PLoS One 8, 6 (2013).

16. Zhang, L. L. et al. Motion sickness: Current knowledge and recent advance. CNS Neurosci. Ther. 22(1), 15-24 (2016).

17. Galvani, C., Ardigò, L. P., Alberti, M., Daniele, F. \& Capelli, C. Physical activity, sleep pattern and energy expenditure in doublehanded offshore sailing. J. Sports Med. Phys. Fitness 55(12), 1480-1487 (2015).

18. Neville, V. et al. America’s Cup yacht racing: Race analysis and physical characteristics of the athletes. J. Sports Sci. 27(9), 915-923 (2009).

19. Bernardi, M. et al. Physiological characteristics of America’s Cup sailors. J. Sports Sci. 25(10), 1141-1152 (2007).

20. Neville, V., Gant, N. \& Folland, J. P. Thermoregulatory demands of Elite Professional America’s Cup Yacht Racing. Scand. J. Med. Sci. Sports 20(3), 475-484 (2010).

21. Mrakic-Sposta, S. et al. Acute effects of triathlon race on oxidative stress biomarkers. Oxid. Med. Cell Longev. 20, 20 (2020).

22. Vezzoli, A. et al. Oxidative stress assessment in response to ultraendurance exercise: Thiols redox status and ROS production according to duration of a competitive race. Oxid. Med. Cell Longev. 20, 16 (2016).

23. Mrakic-Sposta, S. et al. Training effects on ROS production determined by electron paramagnetic resonance in master swimmers. Oxid. Med. Cell Longev. 20, 15 (2015).

24. Zhang, J. et al. ROS and ROS-mediated cellular signaling. Oxid. Med. Cell Longev. 20, 16 (2016).

25. Liguori, I. et al. Oxidative stress and diseases. Oxid. Stress Dis. 20, 757-772 (2012).

26. Elliott, S. N. \& Wallace, J. L. Nitric oxide: A regulator of mucosal defense and injury. J. Gastroenterol. 33(6), 792-803 (1998).

27. Golding, J. F., Bles, W., Bos, J. E., Haynes, T. \& Gresty, M. A. Motion sickness and tilts of the inertial force environment: Active suspension systems vs active passengers. Aviat Sp. Environ. Med. 74(3), 220-227 (2003).

28. Mrakic-Sposta, S. et al. Oxidative stress assessment in breath-hold diving. Eur. J. Appl. Physiol. 119(11-12), 2449-2456. https:// doi.org/10.1007/s00421-019-04224-4 (2019).

29. Mrakic-Sposta, S. et al. Change in oxidative stress biomarkers during 30 days in saturation dive: A pilot study. Int. J. Environ. Res. Public Health 17(19), 1-11 (2020).

30. Mrakic-Sposta, S., Gussoni, M., Montorsi, M., Porcelli, S. \& Vezzoli, A. Assessment of a standardized ROS production profile in humans by electron paramagnetic resonance. Oxid. Med. Cell Longev. 20, 12 (2012).

31. Dorn, L. D., Lucke, J. F., Loucks, T. L. \& Berga, S. L. Salivary cortisol reflects serum cortisol: Analysis of circadian profiles. Ann. Clin. Biochem. 44(3), 281-284 (2007).

32. Bosco, G. et al. Spirometry and oxidative stress after rebreather diving in warm water. Undersea Hyperb. Med. 45(2), 191-198 (2018).

33. Green, L. C. et al. Analysis of nitrate, nitrite, and [15N]nitrate in biological fluids. Anal. Biochem. 126(1), 131-138 (1982).

34. Glantzounis, G., Tsimoyiannis, E., Kappas, A. \& Galaris, D. Uric acid and oxidative stress. Curr. Pharm. Des. 11(32), 4145-4151 (2005).

35. Oldenburg, M. \& Jensen, H. J. Saliva cortisol level as a strain parameter for crews aboard merchant ships. Chronobiol. Int. 36(7), 1005-1012. https://doi.org/10.1080/07420528.2019.1604540 (2019).

36. Main, L. C., Wolkow, A. \& Chambers, T. P. Quantifying the physiological stress response to simulated maritime pilotage tasks. J. Occup. Environ. Med. 59(11), 1078-1083 (2017).

37. Liberzon, J., Abelson, J. L., King, A. \& Liberzon, I. Adaptation Seafaring 33(7), 1023-1026 (2009).

38. Whitson, P. A., Putcha, L., Chen, Y.-M. \& Baker, E. Melatonin and Cortisol assessment of circadian shifts in astronauts before flight. J. Pineal Res. 18(3), 141-147 (1995).

39. Hernández, L. M., Markwald, R. R., Kviatkovsky, S. A., Perry, L. N. \& Taylor, M. K. Morning cortisol is associated with stress and sleep in elite military men: A brief report. Mil. Med. 183(9-10), e255-e259 (2018).

40. Redwine, L., Hauger, R. L., Gillin, J. C. \& Irwin, M. Effects of sleep and sleep deprivation on interleukin-6, growth hormone, cortisol, and melatonin levels in humans. J. Clin. Endocrinol. Metab. 85(10), 3597-3603 (2000).

41. Abedelmalek, S. et al. Effect of time of day and partial sleep deprivation on plasma concentrations of IL-6 during a short-term maximal performance. Eur. J. Appl. Physiol. 113(1), 241-248 (2013).

42. Hayes, L. D., Grace, F. M., Baker, J. S. \& Sculthorpe, N. Exercise-induced responses in salivary testosterone, cortisol, and their ratios in men: A meta-analysis. Sport Med. 45(5), 713-726. https://doi.org/10.1007/s40279-015-0306-y (2015).

43. Lukina, G. I. et al. The oral mucosa status and the correlation between the functional parameters and the level of nitric oxide metabolites in saliva among patients with GERD. Int J Dent. 20, 20 (2020). 
44. Beyazit, F., Türkön, H., Pek, E., Ozturk, F. H. \& Ünsal, M. Elevated circulating nitric oxide levels correlates with enhanced oxidative stress in patients with hyperemesis gravidarum. J. Obstet. Gynaecol. (Lahore) 38(5), 668-673. https://doi.org/10.1080/01443615. 2017.1383371 (2018)

45. Wang, J., Li, J., Yu, M., Wang, Y. \& Ma, Y. An enhanced expression of hypothalamic neuronal nitric oxide synthase in a rat model of simulated transport stress. BMC Vet. Res. 15(1), 1-10 (2019).

46. Zicari, A. et al. Cyclic vomiting syndrome: In vitro nitric oxide and interleukin-6 release by esophageal and gastric mucosa. Dig. Dis. Sci. 46(4), 831-835 (2001).

47. Koch, K. L. Gastric dysrhythmias: A potential objective measure of nausea. Exp. Brain Res. 232(8), 2553-2561 (2014).

48. Philippe, K., Paillard, T., Dubois, R., Maurelli, O. \& Prioux, J. Key performance indicators in Tour de France sailing. J. Sports Sci. 00(00), 1-11. https://doi.org/10.1080/02640414.2020.1851925 (2020).

49. Ghiani, G. et al. Case Study: Physical capacity and nutritional status before and after a single-handed yacht race. Int. J. Sport Nutr. Exerc. Metab. 28(5), 558-563 (2018).

50. Fisher-Wellman, K. \& Bloomer, R. J. Acute exercise and oxidative stress: A 30 year history. Dyn. Med. 8(1), 1-25 (2009).

51. Shi, M. et al. Effects of anaerobic exercise and aerobic exercise on biomarkers of oxidative stress. Environ. Health Prev. Med. 12(5), 202-208 (2007).

52. Bloomer, R. J., Goldfarb, A. H., Wideman, L., McKenzie, M. J. \& Consitt, L. A. Effects of acute aerobic and anaerobic exercise on blood markers of oxidative stress. J. Strength Cond. Res. 19(2), 276-285 (2005).

53. Bloomer, R. J. \& Goldfarb, A. H. Anaerobic exercise and oxidative stress: A review. Can. J. Appl. Physiol. 29(3), 245-263 (2004).

54. Barrios, C. et al. Metabolic muscle damage and oxidative stress markers in an America's Cup yachting crew. Eur. J. Appl. Physiol. 111(7), 1341-1350 (2011).

55. El Assar, M., Angulo, J. \& Rodríguez-Mañas, L. Oxidative stress and vascular inflammation in aging. Free Radic. Biol. Med. 65, 380-401. https://doi.org/10.1016/j.freeradbiomed.2013.07.003 (2013).

56. Siti, H. N., Kamisah, Y. \& Kamsiah, J. The role of oxidative stress, antioxidants and vascular inflammation in cardiovascular disease (a review). Vascul. Pharmacol. 71, 40-56. https://doi.org/10.1016/j.vph.2015.03.005 (2015).

57. Oldenburg, M. Risk of cardiovascular diseases in seafarers. Int. Marit. Health 65(2), 53-57 (2014).

58. Von Katzler, R. et al. Lifestyle behaviour and prevalence of cardiovascular risk factors-a pilot study comparing Kiribati and European seafarers. BMC Public Health 19(1), 1-9 (2019).

59. Eriksson, H. P., Forsell, K. \& Andersson, E. Mortality from cardiovascular disease in a cohort of Swedish seafarers. Int. Arch. Occup. Environ Health. 93(3), 345-353. https://doi.org/10.1007/s00420-019-01486-5 (2020).

60. Murr, C., Widner, B., Wirleitner, B. \& Fuchs, D. Neopterin as a marker for immune system activation. Curr. Drug Metab. 23(3), 175-187 (2002).

61. Hodgson, L. et al. Acute kidney injury associated with endurance events-is it a cause for concern? A systematic review. BMJ Open Sport Exerc. Med. 3, 1 (2017).

62. Cox, P. J. et al. Nutritional ketosis alters fuel preference and thereby endurance performance in athletes. Cell Metab. 24(2), 256-268. https://doi.org/10.1016/j.cmet.2016.07.010 (2016).

63. Sansone, M. et al. Effects of ketone bodies on endurance exercise. Curr. Sports Med. Rep. 17(12), 444-453 (2018).

64. Paoli, A., Bosco, G., Camporesi, E. M. \& Mangar, D. Ketosis, ketogenic diet and food intake control: A complex relationship. Front. Psychol. 6(FEB), 1-9 (2015).

65. Rubini, A. et al. Effects of twenty days of the ketogenic diet on metabolic and respiratory parameters in healthy subjects. Lung 193(6), 939-945 (2015).

66. Otto, B., Riepl, R. L., Klosterhalfen, S. \& Enck, P. Endocrine correlates of acute nausea and vomiting. Auton. Neurosci. Basic Clin. 129(1-2), 17-21 (2006).

67. Meissner, K., Enck, P., Muth, E. R., Kellermann, S. \& Klosterhalfen, S. Cortisol levels predict motion sickness tolerance in women but not in men. Physiol. Behav. 97(1), 102-106. https://doi.org/10.1016/j.physbeh.2009.02.007 (2009).

\section{Acknowledgements}

We thank Extreme Sailing Academy and the skipper S.F. that made this experience possible. A mention should go to the crew that was extremely interested and enthusiastic to take part to the experiment even in those harsh conditions. Thank you S.B., M.W., F.B., A.B. and T.A.G.

\section{Author contributions}

All experiments were performed at university of Padova (Padova, Italy) and National Research Council (Milano, Italy). T.A.G. contributed to the study design, data collection and drafting of the manuscript; A.V. contributed to the data analysis, interpretation and critical review of the manuscript; C.D. contributed to data analysis. M.P. and D.C. contributed to the study design, and critical review of the manuscript. G.B. contributed to the study design, data interpretation, and critical review of the manuscript. S.M.S. contributed to the study design, data analysis, interpretation and drafting of the manuscript. G.B. and S.M.S. confirm that the study objectives and procedures are honestly disclosed. All the authors approved the final version of the manuscript.

\section{Competing interests}

The authors declare no competing interests.

\section{Additional information}

Correspondence and requests for materials should be addressed to T.A.G. or G.B.

Reprints and permissions information is available at www.nature.com/reprints.

Publisher's note Springer Nature remains neutral with regard to jurisdictional claims in published maps and institutional affiliations. 
(c) (i) Open Access This article is licensed under a Creative Commons Attribution 4.0 International cc) License, which permits use, sharing, adaptation, distribution and reproduction in any medium or format, as long as you give appropriate credit to the original author(s) and the source, provide a link to the Creative Commons licence, and indicate if changes were made. The images or other third party material in this article are included in the article's Creative Commons licence, unless indicated otherwise in a credit line to the material. If material is not included in the article's Creative Commons licence and your intended use is not permitted by statutory regulation or exceeds the permitted use, you will need to obtain permission directly from the copyright holder. To view a copy of this licence, visit http://creativecommons.org/licenses/by/4.0/.

(C) The Author(s) 2022 